\title{
Dengue: The Continual Re-Emergence of a Centuries-Old Disease
}

\author{
David M. Morens, ${ }^{1}$ Gregory K. Folkers, ${ }^{1}$ and Anthony S. Fauci ${ }^{1}$ \\ National Institute of Allergy and Infectious Diseases, National Institutes of Health, Bethesda, MD
}

\section{INTRODUCTION}

In 1780, a novel epidemic disease struck Philadelphia, the United States capital at the time. The city's leading physician, Benjamin Rush, a signer of the Declaration of Independence, marveled at the extreme prostration affecting his patients, who experienced high fevers, arthralgias, myalgias, and often an evanescent rash. Because of intense joint and muscle pains, the new disease came to be known as "breakbone fever." Rush observed many patients with extreme post-illness lethargy and depression which he referred to as "dejection of the spirits". He wrote that the new disease might just as well be called "break-heart fever" (Rush 1789). Dengue, as it ultimately was named, has remained with us as a continually re-emerging epidemic disease ever since.

\section{Dengue as an Emerging And Re-emerging INFECTION}

The two-century-old American emergence of dengue is an interesting starting point for considering determinants of emerging infectious diseases that occur today. Unbeknownst to Rush and his contemporaries at the time, dengue is a mosquito-borne infectious disease caused by any of four related flaviviruses designated dengue types 1 through 4 . Dengue viruses are spread by the peri-domestic mosquito Aedes aegypti (also the vector of yellow fever,

Published online: March 1, 2013

Correspondence to: David M. Morens, e-mail: dmorens@niaid.nih.gov which caused a series of fatal epidemics along the East Coast of the United States in the 1790s). In recent decades, dengue has also been transmitted by the related Aedes albopictus, the so-called "Asian tiger mosquito" (Halstead 2007). As early as the eighteenth century, dengue was being spread by human travel and was being introduced into new locales such as Philadelphia by the importation of both mosquitoes and infected crews and passengers arriving from Caribbean islands, with which the young nation of the United States of America had extensive trade relationships. It was strongly suspected that this is how dengue spread, because dengue epidemics in the United States appeared first in East Coast port cities, many of which-like Philadelphia-are in temperate zones that are unable to sustain the mosquito vectors year round. Furthermore, we know several other facts that support this theory of dengue spread: dengue and yellow fever usually began in residential areas close to the docks, only occurred in the summer months, were repeatedly associated with concomitant Caribbean island epidemics, and such epidemics seem to have been most pronounced in places, like Philadelphia, where fresh water was scarce and expensive, leading to onpremise storage of potable water, an ideal environment for Aedes oviposition (egg-depositing).

\section{The Origins of Dengue}

Although we will likely never know for certain where dengue came from originally (or when), the mosquito 
vector and arguably the virus are of African origin and may well have been imported to the Americas with the slave trade (Gould and Solomon 2008). Dengue was not a new disease in 1780; rather it was newly recognized. In retrospect, dengue epidemics probably occurred in the Caribbean islands at least as early as 1635 , when Jean-Baptiste du Tertre reported outbreaks of coup de barre (beating with a stick" (du Tertre 1667; Laughlin et al. 2012)).

To trace the dengue story back to its original American roots, however, we should go back even further to 1492, the year of European discovery of the Americas. At the time, the world population was less than 500 million (it is now more than 7 billion). That relatively small global population was about to embark upon an era of frenetic global travel, movement, and ecologic perturbation that continues unabated, and indeed is still accelerating today. This created the opportunity for geographically and ecologically limited mosquitoes and their viruses to spread globally. Dengue had probably reached Southeast Asia by the late 1700 s, where it found a particularly favorable environment of population crowding, litter, and stored rainwater (ideal for mosquito vector breeding). Because Aedes mosquitoes adapted so well to human crowding, human dwellings, and peoples' habits of creating mosquito-friendly peri-urban habitats, the viruses they took up in human blood meals had access to an almost endless supply of new human hosts. Mosquitoes, which undoubtedly oviposited in pots and shards of water-containing vessels and similar littered debris in the 1700s, adapted easily to discarded tin cans and empty glassware in the 1800s. In the modern era, one of the most important globally dispersed breeding sites for dengue mosquitoes is discarded rubber tires that efficiently collect and retain rainwater.

\section{Current Status of Dengue}

Today, the zone of endemic and epidemic dengue includes the entire tropical/subtropical belt that covers more than 100 nations with a combined population of more than 2.5 billion people (World Health Organization 2012). With 50-100 million new cases and an estimated 20,000 deaths occurring annually, dengue has become arguably the most aggressive re-emerging infectious disease worldwide. Its spread has been directly related to population growth, human movement, and crowding/urbanization. A oncesecondary insect vector, Aedes albopictus, has itself spread globally, expanding the range of dengue cases even further.
Until around 1987, the continental United States was free of this vector. Now Aedes albopictus has become established in at least 27 states, in a range more northerly than that of the classical dengue vector Aedes aegypti. At the same time, potentially pathogenic dengue strains have moved into additional locales and have generated new and often more deadly epidemics in places that were once dengue-free. The year 2012 saw major epidemics in India, Portugal, and Puerto Rico. It is clear that the global dengue problem is getting steadily and progressively worse.

\section{Pathogenesis of Dengue Disease}

Dengue is a disease that results from properties of both the virus (four serotypes and multiple strains, some associated with pathogenicity determinants) and the environment (e.g., mosquito vectors, water storage, and mosquito breeding in objects discarded by humans). Furthermore, in recent decades we have learned that severe forms of dengue disease may also be related to properties of the human immune system, in complex ways that are still not fully understood. Dengue hemorrhagic fever (DHF) and its severe and sometimes fatal form, dengue shock syndrome (DSS), once-rare complications associated with vasculopathy and thrombocytopenia, have in the past 50 years become pandemic (Halstead 2007). DSS, whose pathogenesis is not entirely understood, appears late in the course of an otherwise unremarkable dengue infection when the vascular endothelium is transiently perturbed, allowing extravasation of fluids, electrolytes, and low molecular weight proteins into adjacent tissues; as a result, hypotension and sometimes shock and death occur (Halstead 2007).

Considerable epidemiologic evidence suggests that DHF/DSS has a significant tendency to occur in second dengue infections caused by a different serotype from that causing the first infection, and moreover that certain dengue infection sequences (e.g., dengue type 1 followed by dengue type 2) may be more highly associated with severe disease than other sequences, pointing to the possibility of an immunopathologic mechanism (Halstead 2007; Morens 1994). The leading hypothesis invokes antibody-dependent infection enhancement (ADE), which has been well-characterized in in vitro, although not in vivo, experimental systems (Halstead 2007; Morens 1994). ADE occurs when virus-bound IgG attaches to cell Fc-receptors, resulting in increased viral uptake by cells (Halstead 2007; Morens 1994; 
Murphy and Whitehead 2011). Experimental evidence suggests that dengue ADE could be mediated by crossreactive but non-neutralizing antibody raised by prior infection with a different dengue virus serotype (Halstead 2007; Morens 1994).

It remains unclear why DHF and DSS have become epidemic, and indeed pandemic. Part of the explanation is surely the simple numerical expansion of the human host population. However, this cannot explain completely the rapid rise in incidence. Perhaps particular strains possess determinants that make them more likely to cause DHF/ DSS. Of interest is the observation that when dengue viruses appear and become endemic in new locales, there may be a lag time of a year or more before DHF/DSS appears, raising the possibility of viral adaptation in response to high population immunity, perhaps leading to viruses that escape neutralization to become more "enhanceable" when attached by binding/non-neutralizing antibodies (Morens 1994; Murphy and Whitehead 2011).

\section{Dengue Vaccines}

Currently, no licensed dengue vaccine exists. Several are under preclinical and clinical development (Laughlin et al. 2012; Murphy and Whitehead 2011), but the challenge of making a tetravalent vaccine that cannot enhance natural infection is daunting: the potential for immunopathologic complications and the criteria for assessing the safety of such a vaccine when used in open populations highly exposed to natural infections are both undetermined.

\section{Conclusions}

The centuries-old emergence and the current hyper-reemergence of dengue present a cautionary tale for all who wish to prevent or control infectious diseases. Dengue results from a complicated and dynamic interplay between pathogen, host, and environment, played out on a rapidly changing planet. The prevention strategies tried thus farpublic health measures, mosquito control, and vaccine development-have not been entirely successful. A recent international workshop concluded that the best hope for better prevention and control of dengue is an integrated cross-disciplinary approach involving basic science, public health, vector control, ecology, and many other disciplines (Laughlin et al. 2012). Clearly, dengue is not just a simple re-emerging infectious disease; rather, it is a product of complex ecosystem imbalance. For this reason, dengue likely will be stabilized only by an integrated and multifaceted global response.

\section{About the Art}

"Panic caused by a mosquito in Piccadilly Circus" Illustration from "If Insects Were Bigger" by J. H. KernerGreenwood (1909) The Strand Magazine 38(223): 662. Artist unknown.

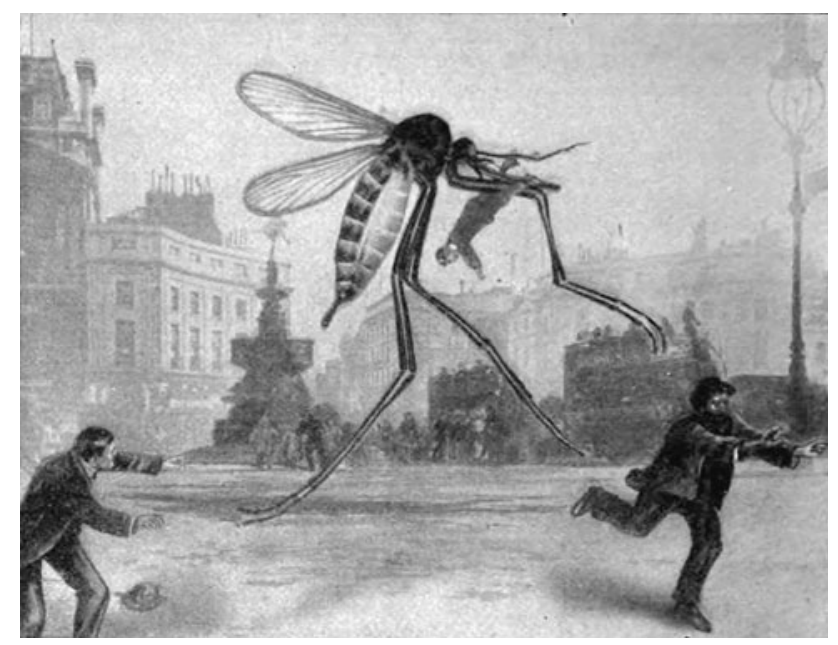

\section{REFERENCES}

du Tertre J-B (1667-1671) Histoire générale des Antilles habitées par les François. Paris: Thomas Jolly

Gould EA, Solomon T (2008) Pathogenic flaviviruses. Lancet 371:500-509

Halstead SB (2007) Dengue. Lancet 370:1644-1652

Laughlin CA, Morens DM, Cassetti MC, Costero-Saint Denis A, San Martin J-L, Whitehead SS, Fauci AS (2012) Dengue research opportunities in the Americas. J Infect Dis 206:1121-1127

Morens DM (1994) Antibody-dependent enhancement of infection and the pathogenesis of viral disease. Clin Infect Dis 19:500512

Murphy BR, Whitehead SS (2011) Immune response to dengue virus and prospects for a vaccine. Annu Rev Immunol 29:587619

Rush B (1789) An account of the bilious remitting fever, as it appeared in Philadelphia, in the summer and autumn of the year 1780. In: Medical Inquiries and Observations, Philadelphia: Prichard and Hall, pp 89-100

World Health Organization (2012) Dengue and severe dengue. Fact sheet No. 117. November 2012. http://www.who.int/ mediacentre/factsheets/fs117/en/ 